\title{
SERUM VASCULAR ENDOTHELIAL GROWTH FACTOR CONCENTRATION IN DOGS DIAGNOSED WITH CHRONIC SUPERFICIAL KERATITIS
}

\author{
Ireneusz BALICKI ${ }^{*}$ and Aleksandra SOBCZYŃSKA-RAK \\ Department and Clinic of Animal Surgery, Faculty of Veterinary Medicine, \\ University of Life Sciences in Lublin, Gleboka 30, 20-612 Lublin, Poland
}

(Received 2 April 2012; accepted 27 September 2012)

\begin{abstract}
The objective of this study was to measure the vascular endothelial growth factor (VEGF) levels in dogs diagnosed with chronic superficial keratitis (CSK). The study was performed on 25 German shepherds (14 males and 11 females, aged between 3 and 11 years). The VEGF levels were determined in blood serum using commercially available enzyme-linked immunosorbent assay (ELISA; Quantikine Canine VEGF Immunoassay, R\&D Systems). The test group of affected German shepherds was subdivided into two subgroups, based on the area of corneal neovascularisation. The first subgroup (9 patients) comprised dogs with neovascularisation observed in 1 to 2 quadrants of the right and left cornea, while the second subgroup (16 patients) comprised dogs with neovascularisation observed in 3 to 4 quadrants of the right and left cornea. The control group comprised 12 clinically healthy German shepherds ( 7 males and 5 females, aged between 3 and 9 years). The results were then statistically analysed by the Mann-Whitney test. The study indicated that the median serum VEGF concentration in healthy dogs was $14.9 \mathrm{pg} / \mathrm{mL}$. The VEGF level observed in sick German shepherds was elevated $(19.5 \mathrm{pg} / \mathrm{mL})$ as compared to the values found in healthy dogs; however, a statistically significant increase in VEGF concentration, as compared to the values observed in healthy dogs, was only noted in the first subgroup, where the median VEGF concentration was $22.0 \mathrm{pg} / \mathrm{mL}$. Elevated serum VEGF concentration was observed in German shepherds diagnosed with CSK. A statistically significant increase in VEGF levels was observed in dogs in the first stage of the disease, i.e. the early stage of neovascularisation.
\end{abstract}

Key words: Vascular endothelial growth factor, VEGF, angiogenesis, chronic superficial keratitis, dog

The cornea is a body part deprived of any blood vessels. Therefore, it can be fully transparent, allowing accurate vision. Many pathological processes in the cornea of both humans and animals, particularly those of chronic nature, in-

*Corresponding author; E-mail: balicki.ireneusz@gmail.com; Phone: 0048 (603) 066-376; Fax: 0048 (81) 524-3808 
volve neovascularisation of the cornea (Gilger et al., 2007; Hosseini and Khalili, 2007; Hosseni and Nejabat, 2007; Balicki and Trbolova, 2010). Neovascularisation is also related to immune-mediated corneal diseases, which include chronic superficial keratitis (CSK) (Stanley, 1988; Bedford and Jones, 2001; Balicki, 2005).

The condition begins with the extension of blood vessels onto the lateral limbus of the temporal quadrant of the cornea. Eventually, neovascularisation extends over a significant part of the cornea, often all of its surface (Balicki, 2005).

Vasculogenesis is a complex, multistage process regulated by a number of stimulants and inhibitors. Vascular endothelial growth factor (VEGF) is one of the chief factors stimulating angiogenesis (Ferrara, 2005). It is secreted by a number of cell types, including fibroblasts, macrophages, activated platelets and neoplastic cells. It is also a 50 times stronger inductor of blood vessel permeability than histamine (Ferrara and Davis-Smyth, 1997). Formation of capillaries is mainly observed in pathological conditions such as local circulation disorders, ischaemia and tissue damage, inflammatory conditions, necrosis and systemic diseases such as diabetes, haemangiopathies, chronic leukaemia, rheumatoid arthritis, psoriasis and cancer (Carmeliet and Jain, 2000; Sobczyńska-Rak, 2009). Vasculogenesis and VEGF expression are regulated by a number of mechanisms, the most important of which seems to be hypoxia. In conditions of lowered oxygen partial pressure, a sudden increase can be observed in the presence of hypoxia inducible factor (HIF-1 $\alpha$ ), which activates the VEGF gene promoter (Rosen, 2002; Stępień-Wyrobiec et al., 2007). VEGF expression can also be stimulated by the cytokines EGF, TGF- $\beta, \mathrm{KGF}, \mathrm{PGF}_{2}$, IGF-1, IL- 1 and IL- 6 . As a strong specific mitogen for endothelial cells, VEGF is an angiogenesis mediator most often determined in blood serum (Sobczyńska-Rak, 2012). VEGF also significantly increases the permeability of endothelium to proteins. It also influences the activation of proteolytic enzymes degrading proteins of the extracellular matrix (including metalloproteins), endothelial cell hyperplasia and migration, as well as the formation of new blood vessels (Ferrara, 2001). VEGF is believed to be responsible for neovascularisation of the cornea which accompanies a number of pathological processes of the eye (Zheng et al., 2001; Andreoli and Miller, 2007). Higher VEGF concentrations have been observed in vascularised corneas when compared with corneas free of lesions (Philipp et al., 2000).

The VEGF receptors -1 (VEGFR1) and -2 (VEGFR2) have been detected in pathological vascular endothelium associated with corneal neovascularisation in dogs (Binder et al., 2011). The results of this study suggest a role of VEGF receptors in both physiological and pathological angiogenesis in canine ocular tissue. It has been observed that VEGF, through its receptors VEGFR1 and VEGFR2, influences keratitis and corneal vascularisation (Philipp et al., 2000). Establishing the role of VEGF and the significance of its concentration for various disease processes is connected to the potential of employing antiangiogenic therapy (Hosseini and Nejabat, 2007). VEGF level was measured in the serum in diabetic 
and nondiabetic cataractous dogs as well as in dogs with intraocular diseases including cataract, primary glaucoma, uveitic glaucoma, aphakic/pseudophakic glaucoma, retinal detachment, lens luxation and intraocular neoplasia (Abrams et al., 2011; Sandberg et al., 2012).

The objective of this study was to determine the concentration of VEGF in the serum of dogs diagnosed with chronic superficial keratitis.

\section{Materials and methods}

The study was performed on 25 German shepherds (14 males and 11 females, aged between 3 and 11 years) diagnosed with CSK. The VEGF levels were determined in blood serum using commercially available enzyme-linked immunosorbent assay (ELISA, Quantikine Canine VEGF Immunoassay, R\&D Systems). At each stage, the determination was performed in accordance with the manufacturer's instructions. Blood sera obtained from affected dogs and from the control animals were frozen at $-75^{\circ} \mathrm{C}$ and transported in dry ice to the 'DIALAB' Laboratory of Medical Analyses in Łódź (a laboratory holding the E1061R quality control certification issued by the Centre for Quality Certification in Laboratory Diagnostics), where the determinations were performed. Test result readings were made with the use of a TECAN microplate reader and Magellan 4.0 software allowing for curvilinear characteristics readings.

The dogs were divided into two groups. Group 1 comprising affected German shepherds was subdivided further into two subgroups based on the extent of neovascularisation on the corneal surface. The first subgroup (IA, $n=9$ patients) comprised dogs in which the neovascularisation covered between 1 and 2 quadrants of the right and left cornea, while in the second subgroup (IB, $\mathrm{n}=16$ patients) neovascularisation covered between 3 and 4 quadrants of the right and left cornea. The control group comprised 12 clinically healthy German shepherds ( 7 males and 5 females, aged between 3 and 9 years). The obtained results were statistically analysed. The Kolmogorov-Smirnov test did not indicate normal distribution of the results, therefore the statistical analysis was based on the MannWhitney test. The correlation between the dogs' ages and the VEGF levels was evaluated using Pearson's correlation. Probability values of $\mathrm{P}<0.05$ were considered significant.

\section{Results}

The VEGF concentrations determined in the different groups are presented in Table 1. VEGF concentrations obtained in clinically healthy German shepherds and German shepherds diagnosed with CSK are presented in Figs 1-4. As compared to concentrations found in healthy dogs, elevated VEGF levels were 
measured in dogs suffering from CSK. The highest VEGF concentrations ( $\mathrm{P}<$ 0.05 ) were observed in dogs in the early stage of neovascularisation (Figs 5a, b), while the lowest values were obtained in dogs in which neovascularisation covered no less than 3 quadrants of the right or left cornea (Figs 6a, b). A positive correlation was found between age and VEGF levels (Pearson's correlation test; $\left.\mathrm{r}_{\mathrm{xy}}=0.71, \mathrm{P}<0.01\right)$ in clinically healthy German shepherds. There was no significant correlation between age and VEGF levels (Pearson's correlation test; $\left.r_{x y}=0.04, P>0.05\right)$ in German shepherds diagnosed with CSK.

Table 1

Serum vascular endothelial growth factor (VEGF) concentrations in clinically healthy German shepherds and in German shepherds diagnosed with chronic superficial keratitis

\begin{tabular}{lcc}
\hline \multirow{2}{*}{ Groups } & \multicolumn{2}{c}{ VEGF concentration $(\mathrm{pg} / \mathrm{mL})$} \\
\cline { 2 - 3 } & Median values & Minimum and maximum values \\
\hline Control group & 14.9 & $3.6-29.6$ \\
Group I & 19.54 & $9.1-72.5$ \\
Group IA & $22.03^{*}$ & $12.0-72.5$ \\
Group IB & 17.82 & $9.1-48.3$ \\
\hline
\end{tabular}

*Statistically significant difference at $\mathrm{P}<0.05$; Group I: clinically healthy dogs; Group IA: patients with neovascularisation covering 1-2 quadrants of the left and right cornea; Group IB - patients with neovascularisation covering 3-4 quadrants of the left and right cornea

\section{Discussion}

Angiogenesis and the influence of VEGF on the formation of pathological blood vessels have been thoroughly researched and confirmed in a number of clinical studies pertaining to disease processes in both humans and animals. Attempts have also been made to determine the influence of VEGF on angiogenesis in diseases of the eyeball (Philipp et al., 2000; Shakiba et al., 2009). The studies of Abrams et al. (2011) and Sandberg et al. (2012) did not find an association between aqueous humour/plasma VEGF levels and intraocular diseases of dogs. VEGF concentration of the aqueous humour was significantly elevated in intraocular diseases such as primary glaucoma, uveitic glaucoma, aphakic/ pseudophakic glaucoma, retinal detachment, lens luxation and intraocular neoplasia, except for primary diabetic cataracts (Sandberg et al., 2012). In this study, VEGF concentration in the aqueous humour exceeded that of the blood plasma. Sandberg et al. (2012) suggest that aqueous humour VEGF levels in these cases are unlikely to be related to circulating VEGF levels and indicate intraocular VEGF production. In its physiological state, the cornea is non-vascularised, which ensures utmost transparency and facilitates the performance of functions 
of the organ of sight. The lack of blood vessels in a healthy cornea is due to low levels of angiogenic factors and high levels of antiangiogenic factors. Any disturbance of that balance can lead to vessel formation occurring in the cornea (Chang et al., 2001). The present study indicated elevated VEGF serum concentrations in dogs suffering from CSK. VEGF concentration was statistically significantly higher $(\mathrm{P}<0.05)$ in dogs in the early stage of the disease, with a median value of $22.03 \mathrm{pg} / \mathrm{mL}(\mathrm{P}<0.05)$ and a peak concentration of $72.5 \mathrm{pg} / \mathrm{mL}$. In comparison, in healthy dogs the maximum median was $29.6 \mathrm{pg} / \mathrm{mL}$. Elevated serum concentrations of an angiogenic factor such as VEGF may indicate its importance in the process of intensive blood vessel formation observed in the early stages of CSK.

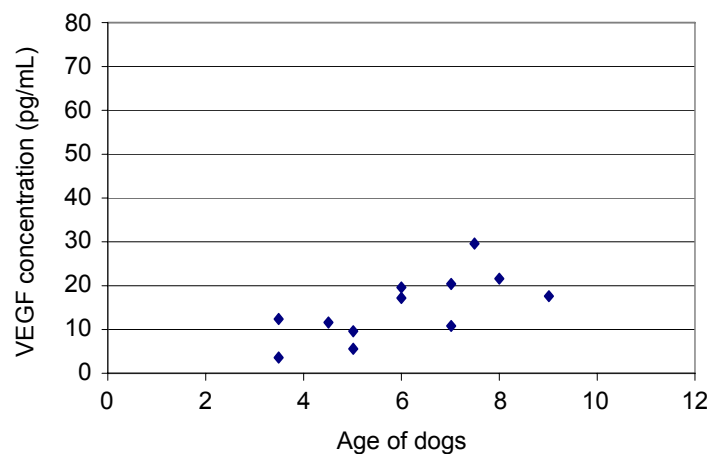

Fig. 1. Vascular endothelial growth factor (VEGF) concentration in healthy dogs (control group)

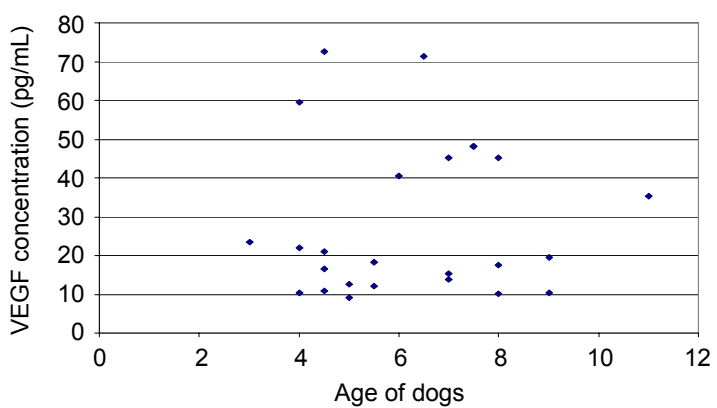

Fig. 2. Vascular endothelial growth factor (VEGF) concentration in dogs diagnosed with chronic superficial keratitis (CSK)

VEGF is a factor stimulating the formation of blood vessels in both physiological and pathological processes of the organism. Therefore, serum VEGF concentrations in healthy animals can be anywhere between $1 \mathrm{pg} / \mathrm{mL}$ and even $40.1 \mathrm{pg} / \mathrm{mL}$ (Clifford et al., 2001; Clifford et al., 2002; Troy et al., 2006; Abrams et al., 2011; Aresu et al., 2012). Clifford et al. (2002) conducted a study on dogs suffering from tumours and compared the VEGF concentrations of healthy and 
diseased animals. They observed that in the control group of healthy animals, the VEGF levels were between 1 and $18 \mathrm{pg} / \mathrm{mL}$. On the other hand, Aresu et al. (2012) reported that the mean growth factor concentration observed in healthy dogs was $40.1 \mathrm{pg} / \mathrm{mL} \pm 10.4 \mathrm{pg} / \mathrm{mL}$. In the authors' own research, the serum VEGF concentration in healthy dogs was observed to oscillate between $3.6 \mathrm{pg} / \mathrm{mL}$ and $29.6 \mathrm{pg} / \mathrm{mL}$, which with the median of $14.9 \mathrm{pg} / \mathrm{mL}$ is similar to values reported by other researchers (Clifford et al., 2001; Clifford et al., 2002) and in line with natural changeability. The authors created a control group comprising only German shepherds and carried on to compare the VEGF levels of diseased German shepherds to levels found in healthy dogs of the same breed.

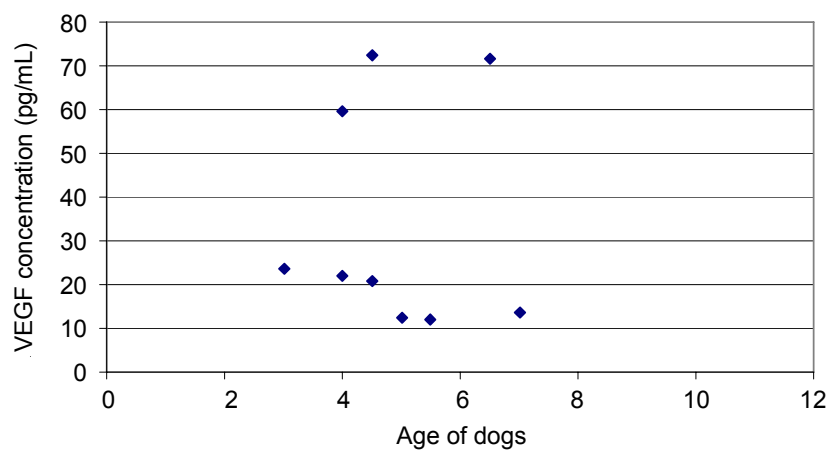

Fig. 3. VEGF concentration in dogs diagnosed with CSK - patients with neovascularisation covering between 1 and 2 quadrants of the left and right cornea (subgroup IA)

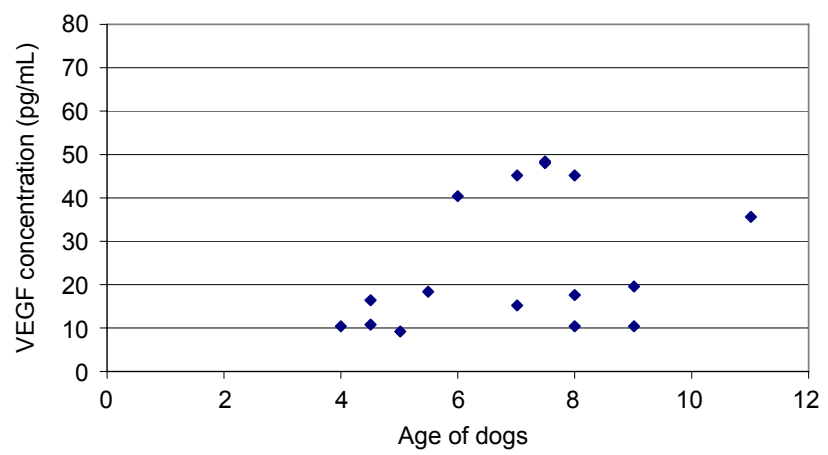

Fig. 4. VEGF concentration in dogs diagnosed with CSK - patients with neovascularisation covering between 3 and 4 quadrants of the left and right cornea (subgroup IB)

Abrams et al. (2011) demonstrated that the VEGF level may depend on the age and gender of the animal: they observed that in older dogs (10-15 years old) the VEGF levels were higher than those measured in young animals. Our study also showed a positive correlation $(\mathrm{P}<0.01)$ between age and VEGF lev- 
els in clinically healthy German shepherds, with the VEGF levels being higher in older dogs. Chronic superficial keratitis is most often diagnosed in dogs between 4 and 8 years of age (Balicki, 2005). In the authors' own research, $84 \%$ of the dogs diagnosed with CSK were between 4 and 8 years of age and, with one exception, all dogs were under 10 years old. Statistical analysis revealed no significant correlation $(\mathrm{P}>0.05)$ between age and VEGF levels in dogs suffering from CSK. It has been observed that the VEGF levels of both the blood plasma and the serum were higher in female dogs than in male animals (Abrams et al., 2011). In contrast, in their study on Alaskan sled dogs, Kemp et al. (2005) found higher mean levels in male $(21.7 \mathrm{pg} / \mathrm{mL})$ than in female dogs $(17.7 \mathrm{pg} / \mathrm{mL})$, and the VEGF concentrations of healthy Alaskan sled dogs oscillated between 9 and $34 \mathrm{pg} / \mathrm{mL}$. Considering this, the present study was conducted on 14 male and 11 female dogs affected with CSK, while the control group of healthy animals comprised 7 male and 5 female animals.
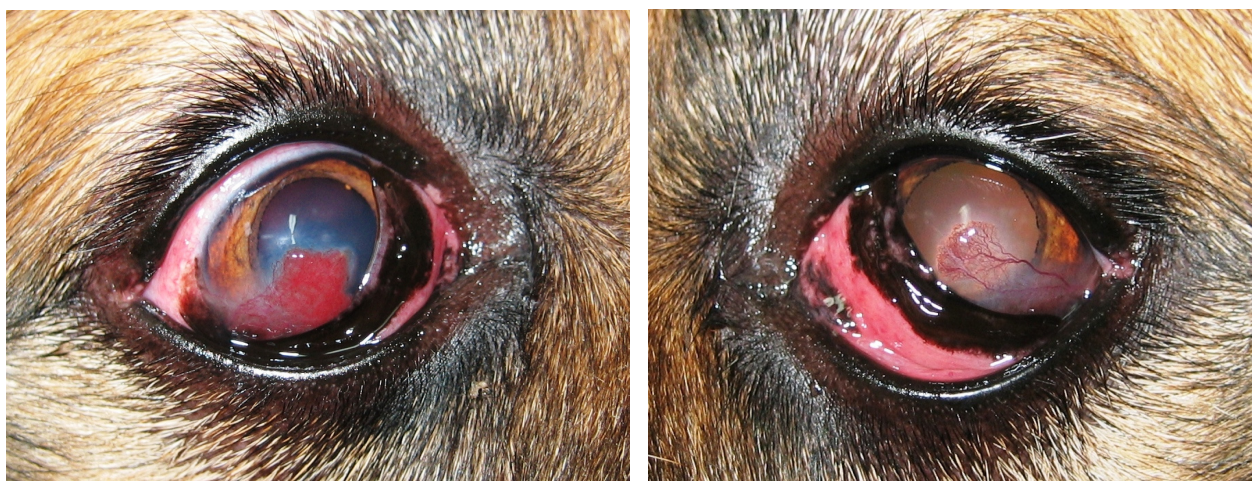

Fig. $5 a$ and $5 b$. Early stages of chronic superficial keratitis (4-year-old male, subgroup IA, VEGF concentration $59.6 \mathrm{pg} / \mathrm{mL}$ )
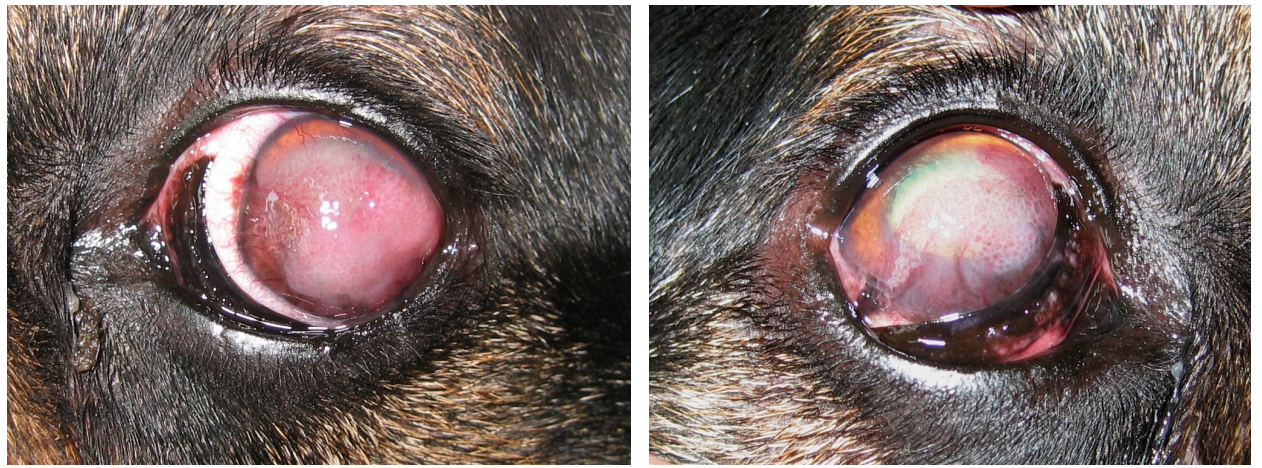

Fig. $6 a$ and $6 b$. Advanced stages of chronic superficial keratitis (4-year-old male, subgroup IB, VEGF concentration $10.2 \mathrm{pg} / \mathrm{mL}$ ) 
There is abundant evidence to confirm the participation of VEGF in corneal neovascularisation (Chen et al., 2002; Gan et al., 2004; Zhou et al., 2006). It has been demonstrated that VEGF expression is significantly higher in vascularised corneas as compared to healthy ones. The main sources of growth factor in the cornea include macrophages, infiltrating leukocytes, the corneal epithelium and stromal cells. VEGF overexpression in the cornea promotes several stages of angiogenesis, i.e. proteolytic reactions, proliferation, migration and formation of vessels from endothelial cells (Shakiba et al., 2009). The pathomechanism of neovascularisation in cases of CSK remains undetermined. Philipp et al. (2000) observed the presence of receptors characteristic of VEGF in the cornea. The research demonstrated elevated VEGF levels in the blood serum of dogs diagnosed with CSK. It is assumed that the most important sources of VEGF in chronic inflammatory conditions of the cornea could be macrophages located mainly in the stroma of inflamed and vascularised corneas. It is believed that macrophages may play a key role in the pathogenesis of pathological neovascularisation of the cornea by secreting VEGF directly and by producing other angiogenic factors which may also trigger VEGF production by other cells, e.g. epithelial cells and keratocytes (Philipp et al., 2000). Williams (1999) demonstrated the presence of macrophages in the corneas of dogs suffering from CSK.

Vasculogenesis of the cornea occurs in dogs in relation to various ocular pathological processes, including inflammations or injuries of the cornea, but also in connection with immune-mediated diseases such as CSK. Therefore, in certain clinical cases there may be a need to monitor the possibility of neovascularisation by determining the concentration of VEGF. In our own research on German shepherd dogs diagnosed with CSK, significant VEGF overexpression was observed in the early stage of the disease, at the beginning of neovascularisation, which may be related to intensive blood vessel ingrowth into the cornea. On the other hand, in dogs in advanced stages of the disease, the VEGF levels were elevated but the difference was statistically insignificant and the concentrations were lower than those observed in the early stage.

The main environmental factor which significantly influences the development of CSK is ultraviolet radiation (Chavkin et al., 1994; Gilger et al., 2007). It is believed that ultraviolet radiation may influence photochemical changes of the cornea, leading to the creation of free radicals and formation of antigens (Campbell et al., 1975; Scotto et al., 1988; Stanley, 1988). Therefore, exposure to ultraviolet radiation is a factor predisposing dogs to CSK (Bedford and Jones, 2001). It is believed that both UV radiation itself and the related hypoxia contribute to the activation of genes in macrophages, neutrophils and other cells, including those coding for VEGF (Youn et al., 1988; Tacchini et al., 1999). It was found that UV radiation induces VEGF expression (Li et al., 2006). Therefore, it should be assumed that UV radiation may play a role in the demonstrated elevation of VEGF levels in dogs suffering from CSK. 
One of the research interests pertains to the possibility of influencing the modulation of angiogenesis with reactive oxygen species (Abid et al., 2001; Colavitti et al., 2002). Research has demonstrated that VEGF stimulates endothelial cells to produce peroxynitrite. Peroxynitrite as a second messenger impinges the activation of multiple signalling pathways leading to endothelial migration, proliferation, and tube formation in vitro and angiogenesis in vivo (El-Remessy et al., 2007). Research conducted by Sreekumar et al. (2006) demonstrated that oxidative stress induced by glutathione depletion leads to the induction of VEGF-A gene expression and VEGFR-1 and VEGFR-2 receptors in retinal pigment epithelial cells. The study showed that cellular redox status plays an important role in VEGF regulation in retinal pigment epithelial cells (Sreekumar et al., 2006). In dogs suffering from CSK, oxidative stress has been reported. Disorders of the antioxidative system and the increased concentrations of protein and lipid peroxidation products observed in dogs suffering from CSK indicated the participation of reactive oxygen species in the disease process (Balicki, 2005). It can be assumed that reactive oxygen species may play a role in the observed elevation of VEGF concentrations in dogs suffering from CSK, but the actual nature of that relationship remains to be determined in the course of future research.

Based on the results of this study, it can be hypothesised that VEGF plays a significant role in corneal neovascularisation in dogs suffering from CSK, while another factor is responsible for maintaining the correct morphology of the blood vessel network formed during angiogenesis. However, further clinical research is needed to confirm this phenomenon.

\section{References}

Abid, M. R., Tsai, J. C., Spokes, K. C., Deshpande, S. S., Irani, K. and Aird, W. C. (2001): Vascular endothelial growth factor induces manganese-superoxide dismutase expression in endothelial cells by a Rac1-regulated NADPH oxidase-dependent mechanism. FASEB J. 15, $2548-2550$.

Abrams, K. L., Stabila, P. F., Kauper, K. and Elliott, S. (2011): Vascular endothelial growth factor in diabetic and nondiabetic canine cataract patients. Vet. Ophthalmol. 14, 93-99.

Andreoli, C. M. and Miller, J. W. (2007): Anti-vascular endothelial growth factor therapy for ocular neovascular disease. Curr. Opin. Ophthalmol. 18, 502-508.

Aresu, L., Aricò, A., Comazzi, S., Gelain, M. E., Riondato, F., Mortarino, M., Morello, E., Stefanello, D. and Castagnaro, M. (2012): VEGF and MMP-9: biomarkers for canine lymphoma. Vet. Comp. Oncol. 10 April 2012, doi: 10.1111/j. 1476-5829.2012.00328.x Epub ahead of print.

Balicki, I. (2005): Research on Diagnostics and Treatment of Chronic Superficial Keratitis in Dogs. Postdoctoral Dissertation, WAR, Lublin, 204, 62-67.

Balicki, I. and Trbolova, A. (2010): Clinical evaluation of tacrolimus eye drops for chronic superficial keratitis treatment in dogs. Bull. Vet. Inst. Pulawy 54, 251-258.

Bedford, P. G. and Jones, G. (2001): Abnormal appearance. In: Peiffer, R. L. and Petersen-Jones, S. M. (eds) Small Animal Ophthalmology: A Problem-oriented Approach. 3rd edition. W. B. Saunders, London. p. 88. 
Binder, D. R., Herring, I. P., Zimmerman, K. L., Pickett, J. Ph. and Huckle, W. R. (2011): Expression of vascular endothelial growth factor receptor-1 and -2 normal and diseased canine eyes. Vet. Ophthalmol. 15, 223-230.

Campbell, L. H., Okuda, H. K., Lipton, D. E. and Reed, C. (1975): Chronic superficial keratitis in dogs: detection of cellular hypersensitivity. Am. J. Vet. Res. 36, 669-671.

Carmeliet, P. and Jain, R. K. (2000): Angiogenesis in cancer and other diseases. Nature 407, 249-257.

Chang, J. H., Gabison, E. E., Kato, T. and Azar, D. T. (2001): Corneal neovascularization. Curr. Opin. Ophthalmol. 12, 242-249.

Chavkin, M. J., Roberts, M. S., Salman, M. D., Severin, G. A. and Scholten, N. J. (1994): Risk factors for development of chronic superficial keratitis in dogs. J. Am. Vet. Med. Assoc. 204, $1630-1634$.

Chen, J., Liu, W., Liu, Z., Chen, L., Huang, T. and Zhen, H. (2002): Expression of vascular endothelial growth factor and its receptors (FLT-1) in morbid human corneas and investigation of its clinical importance. Yan Ke Xue Bao 18, 203-207.

Clifford, C. A., Hughes, D., Beal, M. W., Henry, C. J., Drobatz, K. J. and Sorenmo, K. U. (2002): Vascular endothelial growth factor concentrations in body cavity effusions in dogs. J. Vet. Intern. Med. 16, 164-168.

Clifford, C. A., Hughes, D., Beal, M. W., Mackin, A. J., Henry, C. J., Shofer, F. S. and Surenmo, K. U. (2001): Plasma vascular endothelial growth factor (VEGF) concentrations in normal dogs and dogs with hemangiosarcoma. J. Vet. Intern. Med. 15, 131-135.

Colavitti, R., Pani, G., Bedogni, B., Anzevino, R., Borrello, S., Waltenberger, J. and Galeotti, T. (2002): Reactive oxygen species as downstream mediators of angiogenic signaling by vascular endothelial growth factor receptor-2/KDR. J. Biol. Chem. 277, 3101-3108.

El-Remessy, A. B., Al-Shabrawey, M., Platt, D. H., Bartoli, M., Behzadian, M. A., Ghaly, N., Tsai, N., Motamed, K. and Caldwell, R. B. (2007): Peroxynitrite mediates VEGF's angiogenic signal and function via a nitration-independent mechanism in endothelial cells. FASEB J. 21, 2528-2539.

Ferrara, N. (2005): The role of VEGF in the regulation of physiological and pathological angiogenesis. EXS 94, 209-231.

Ferrara, N. (2001): Role of vascular endothelial growth factor in regulation of physiological angiogenesis. Am. J. Physiol. Cell Physiol. 280, 1358-1366.

Ferrara, N. and Davis-Smyth, T. (1997): The biology of vascular endothelial growth factor. Endocr. Rev. 18, 4-25.

Gan, L., Fagerholm, P. and Palmblad, J. (2004): Vascular endothelial growth factor VEGF and its receptor VEGFR-2 in the regulation of corneal neovascularization and wound healing. Acta Ophthalmol. Scand. 82, 557-563.

Gilger, B. C., Bentley, E. and Ollivier, F. J. V. (2007): Diseases and surgery of the canine cornea and sclera. In: Gelatt, K. N. (ed.) Veterinary Ophthalmology. 4th edition. Blackwell Publishing, Ames. pp. 722-723.

Hosseini, H. and Khalili, M. R. (2007): Therapeutic potential of bevacizumab (Avastin) in herpetic stromal keratitis. Med. Hypotheses 69, 568-570.

Hosseini, H. and Nejabat, M. (2007): A potential therapeutic strategy for inhibition of corneal neovascularization with new anti-VEGF agents. Med. Hypotheses 69, 799-801.

Kemp, S. W., Reynolds, A. J. and Duffy, L. K. (2005): Gender differences in baseline levels of Vascular Endothelial Growth Factor in plasma of Alaskan Sled dogs. Am. J. Biochem. Biotechnol. 1, 111-114.

Li, Y., Bi, Z., Yan, B. and Wan, Y. (2006): UVB radiation induces expression of HIF-1alpha and VEGF through the EGFR/PI3K/DEC1 pathway. Int. J. Mol. Med. 18, 713-719.

Philipp, W., Speicher, L. and Humpel, Ch. (2000): Expression of Vascular Endothelial Growth Factor and its receptors in inflamed and vascularized human corneas. Invest. Ophthalmol. Vis. Sci. 41, 2514-2522. 
Rosen, L. S. (2002): Clinical experience with angiogenesis signaling inhibitors: focus on vascular endothelial growth factor (VEGF) blockers. Cancer Control 9, 36-44.

Sandberg, C. A., Herring, I. P., Huckle, W. R., LeRoith, T., Pickett, J. P. and Rossmeisl, J. H. (2012): Aqueous humor vascular endothelial growth factor in dogs: association with intraocular disease and the development of pre-iridal fibrovascular membrane. Vet. Ophthalmol. 15, 21-30.

Scotto, J., Cotton, G., Urbach, F., Berger, D. and Fears, T. (1988): Biologically effective ultraviolet radiation: surface measurements in the United States, 1974 to 1985. Science 239, 762-764.

Shakiba, Y., Mansouri, K., Arshadi, D. and Rezaei, N. (2009): Corneal neovascularization: Molecular events and therapeutic options. Recent Pat. Inflamm. Allergy Drug Discov. 3, 221-231.

Sobczyńska-Rak, A. (2009): Correlation between plasma VEGF and angiogenesis of skin and subcutaneous tissue cancer in dogs. Bull. Vet. Inst. Pulawy 53, 503-506.

Sobczyńska-Rak, A. (2012): The role of VEGF in process of neovasculogenesis. In: Ran, S. (ed.) Tumor Angiogenesis. Intech, Rijeka, Croatia. pp. 181-196.

Sreekumar, P. G., Kannan, R., de Silva, A. T., Burton, R., Ryan, S. J. and Hinton, D. R. (2006): Thiol regulation of vascular endothelial growth factor-A and its receptors in human retinal pigment epithelial cells. Biochem. Biophys. Res. Commun. 346, 1200-1206.

Stanley, R. G. (1988): Superficial stromal keratitis in the dog. Aust. Vet. J. 65, 321-323.

Stępień-Wyrobiec, O., Wyrobiec, G., Rokicki, W. and Harabin-Słowińska, M. (2007): Vascular endothelial growth factor (VEGF) - a regulator of angiogenesis. Ann. Acad. Med. Silesian. 61, 152-160.

Tacchini, L., Bianchi, L., Bernelli-Zazzera, A. and Cairo, G. (1999): Transferrin receptor induction by hypoxia. HIF-1-mediated transcriptional activation and cell-specific post-transcriptional regulation. J. Biol. Chem. 274, 24142-24146.

Troy, G. C., Huckle, W. R., Rossmeisl, J. H., Panciera, D., Lanz, O., Robertson, J. L. and Ward, D. L. (2006): Endostatin and vascular endothelial growth factor concentrations in healthy dogs, dogs with selected neoplasia, and dogs with nonneoplastic diseases. J. Vet. Intern. Med. 20, 144-150.

Williams, D. L. (1999): Histological and immunohistochemical evaluation of canine chronic superficial keratitis. Res. Vet. Sci. 67, 191-195.

Youn, J. I., Gange, R. W., Maytum, D. and Parrish, J. A. (1988): Effect of hypoxia on sunburn cell formation and inflammation induced by ultraviolet radiation. Photodermatol. 5, 252-256.

Zheng, M., Deshpande, S., Lee, S., Ferrara, N. and Rouse, B. T. (2001): Contribution of vascular endothelial growth factor in the neovascularization process during the pathogenesis of herpetic stromal keratitis. J. Virol. 75, 9828-9835.

Zhou, L. H., Xing, Y. Q., Chen, C. L. and Wang, D. W. (2006): Antisense vascular endothelial growth factor suppressed corneal neovascularization in rats. Chin. J. Ophthalmol. 42, 426-430. 